\title{
Assessing legend designs for coordinated and multiple views geovisualizations
}

\author{
Izabela Gołębiowska $^{\mathrm{a}, *}$, Tomasz Opach ${ }^{\mathrm{b}}$, Arzu Çöltekin ${ }^{\mathrm{c}}$ \\ ${ }^{a}$ University of Warsaw, Faculty of Geography and Regional Studies, Department of Geoinformatics, Cartography and Remote \\ Sensing,i.golebiowska@uw.edu.pl, \\ ${ }^{b}$ Norwegian University of Science and Technology, Faculty of Social and Educational Sciences, Department of Geography, \\ tomasz.opach@ntnu.no, \\ ${ }^{c}$ University of Applied Sciences and Arts Northwestern Switzerland, School of Engineering, Institute of Interactive Technologies, \\ arzu.coltekin@fhnw.ch
}

* Corresponding author

Keywords: coordinated and multiple views, layout design, legend, user study

\begin{abstract}
:
Coordinated and multiple views (CMV) geovisualizations are the core of geovisual analytics solutions. They consist of several dynamically connected data displays presenting spatial data by means of different visualization types, e.g., maps, graphs, plots, charts (Roberts 2007). Such CMV geovisualizations are to support exploration of large data sets through interactive functions with the help of diverse insights offered by the visualization types included in the layout. Kraak $e t$ al. (2020) place CMVs into the category of analytical dashboards and describe them as tools with coordinated interaction between views for generating previously unknown insights into patterns and anomalies across a range of datasets.

Even though CMV geovisualizations do enable in-depth insights into large data sets, they might be perceived as difficult and discouraging for users due to the visual complexity of the information-rich dashboard. Therefore, explanatory elements that help a user understand how to work with a CMV display, i.e. decode the symbology (e.g., map legend), and explain how to explore the data (e.g., filter, select, change the presented subsets of data) are of high value. When designing a CMV layout, these legend-like guiding display elements should be designed carefully, and ideally, guided by empirical evidence. Legend design of various visualization types has been subject to thorough analyses in previous work: Map legends are discussed in every cartography handbook, and empirical research has repeatedly addressed legend design in maps (e.g., Çöltekin et al. 2016, Edler 2020, Gołębiowska 2015). However, CMV geovisualizations are different from maps in a sense that they consist of several views, and each of them requires its own legend or legend-like explanatory display elements. Moreover, all of them should be designed and organised in a meaningful way, consistent across the entire interface. In a CMV, explanatory elements can be organized either as separate multiple legends located within (or next to) each corresponding view, or as a merged legend, with elements from all views grouped in one place. Both design approaches have strengths. The first solution (separate views) provides advantages due to the proximity of the legends to the explained views (and complies with e.g. Gestalt law of proximity). In turn, the second approach (a single, merged legend) follows the recommendation of Schnürer et al. (2015) for functional grouping and clustering tools instead of distributing across the layout. Although these are contradictory design options, to the best of our knowledge, these two approaches lack comparative studies that would provide practitioners, CMV designers and map makers with empirical evidence that would help them gain a good understanding which approach is helpful for and desired by CMV users. To do so, we have implemented both approaches in a CMV tool which supports examination of insurance compensation payments due to losses caused by various natural hazards, and visualizes spatio-temporal data. Our intention was to examine which of the two approaches facilitates information processing better for the users. Both interfaces consist of three views: a thematic map, a bar graph and a table. They differ only with location and organization of explanatory elements within a layout. To comparatively evaluate the two legend design approaches, we conducted an empirical user study with mixed factorial design. The main independent variable in the experiment was the two CMV layouts of the same tool: with multiple, and with a merged legend. As dependent variables, we collected both objective (response time, response accuracy, eye-tracking metrics), and subjective (preference, response confidence) metrics. Layout and questions were within-subject, whereas a free exploration task was between-subject.
\end{abstract}

In total 42 participants voluntarily took part in the study (aged 23-30, 52\% female, $48 \%$ male). All of them were graduate students in cartography, holding at least a Bachelor's degree, at the Faculty of Geography and Regional Studies, University 
of Warsaw, Poland. They were experienced in working with interactive maps, but not familiar with the tested tool. The participants used the tool, elicited necessary information and answered eight questions, representing four task types classified by Knapp (1995): Identify, locate, compare, and associate. The tasks were designed to refer to configurations corresponding to a seminal triad framework by Peuquet (1994): (1) Explore objects that are present at a given location at a given time (when + where -> what); (2) Explore times that a given object occupied a given location (where + what $->$ when); (3) Explore locations occupied by a given object at a given time (when + what $->$ where). The choice and specification of tasks were supported by semi-structured interviews with four experts from the insurance industry arranged ahead of the empirical study. Exemplary task required answering if an investment in a particular year resulted with a visible change of insurance data in the following years. In order to investigate the spontaneous learning of the tested tool, participants conducted a free exploration task at the beginning of the study. The collected empirical data is then analysed to compare differences between participants' performance and subjective responses while using the two layouts both in terms of effectiveness and information processing strategies.

With the work-in-progress study reported here, we hope to contribute to an increased understanding of how legends and legend-like display elements are used in the context of CMV environments. The knowledge gained from this empirical study will contribute towards developing design recommendations for CMVs that effectively support users in decision making with visual analytics tools.

\section{Acknowledgements}

This work was supported by the National Science Centre, Poland under the grant no. UMO-2018/31/D/HS6/02770.

\section{References}

Çöltekin, A., Brychtova, A., Griffin, A., Robinson, A., Imhof, M., Pettit, C. 2016. Perceptual complexity of soil-landscape maps: a user evaluation of color organization in legend designs using eye tracking. International Journal of Digital Earth, Vol. 10, No. 6, pp. 560-581.

Edler, D., Keil, J., Tuller, M.-C., Bestgen, A.-K., \& Dickmann, F. 2020. Searching for the 'Right' Legend: The Impact of Legend Position on Legend Decoding in a Cartographic Memory Task. The Cartographic Journal, Vol. 57, No. 1, pp. 6-17.

Gołębiowska, I,. 2015. Legend Layouts for Thematic Maps: A Case Study Integrating Usability Metrics with the Thinking Aloud Method. The Cartographic Journal, Vol. 52, No. 1, pp. 28-40.

Knapp, L., 1995. A Task Analysis Approach to the Visualization of Geographic Data. In: Cognitive Aspects of HumanComputer Interaction for Geographic Information Systems, Springer Netherlands, pp. 355-371.

Kraak, M.-J., Roth, R.E., Ricker, B., Kagawa, A., Sourd, L.G., 2020. Mapping for a Sustainable World. United Nations, International Cartographic Association.

Peuquet, D. J., 1994. It's About Time: A Conceptual Framework for the Representation of Temporal Dynamics in Geographic Information Systems. Annals of the Association of American Geographers, Vol. 84, No. 3, pp. 441-461.

Roberts, J.C., 2007. State of the art: coordinated \& multiple views in exploratory visualization. In: Proc., Fifth International Conference on Coordinated and Multiple Views in Exploratory Visualization, pp. 61-71.

Schnürer, R., Sieber, R., Çöltekin, A., 2015. The Next Generation of Atlas User Interfaces: A User Study with "Digital Natives." In: Modern Trends in Cartography, pp. 23-36. 